\title{
Effects of Nonsurgical Periodontal Therapy on Clinical Response, Microbiological Profile, and Glycemic Control in Malaysian Subjects with Type 1 Diabetes
}

\author{
Samira Mukhtar Buzinin, ${ }^{1}$ Aied Mohammed Alabsi, ${ }^{2}$ Alexander Tong Boon Tan, ${ }^{3}$ \\ Vui King Vincent-Chong, ${ }^{4}$ and Dasan Swaminathan ${ }^{1}$ \\ ${ }^{1}$ Department of Restorative Dentistry, Faculty of Dentistry, University of Malaya, 50603 Kuala Lumpur, Malaysia \\ ${ }^{2}$ Oral Cancer Research and Coordinating Center, Department of Oral Biology and Biomedical Science, Faculty of Dentistry, \\ University of Malaya, 50603 Kuala Lumpur, Malaysia \\ ${ }^{3}$ Department of Endocrinology, University of Malaya Medical Center, 50603 Kuala Lumpur, Malaysia \\ ${ }^{4}$ Oral Cancer Research and Coordinating Center, Department of Oro-Maxillofacial and Medical Science, \\ Faculty of Dentistry, University of Malaya, 50603 Kuala Lumpur, Malaysia
}

Correspondence should be addressed to Dasan Swaminathan; dasans@um.edu.my

Received 29 March 2014; Revised 1 July 2014; Accepted 10 July 2014; Published 23 July 2014

Academic Editor: Reginaldo Bruno Gonçalves

Copyright (C) 2014 Samira Mukhtar Buzinin et al. This is an open access article distributed under the Creative Commons Attribution License, which permits unrestricted use, distribution, and reproduction in any medium, provided the original work is properly cited.

\begin{abstract}
The association between diabetes mellitus and chronic periodontal disease has long been established. Most of the researches linking these two very common chronic diseases were based on type 2 diabetes mellitus and chronic periodontal disease. However, this study was conducted to investigate the association between type 1 diabetes and chronic periodontal disease in Malaysian subjects. Forty-one Malaysian subjects, of which 20 subjects were type 1 diabetics and with chronic periodontal disease (test group) and 21 subjects with only chronic periodontal disease (control group), were included in the study. Periodontal parameters and plaque samples for microbiological evaluation were done at baseline, 2 and 3 months after nonsurgical periodontal therapy. Blood samples were taken from only the test group and evaluated for HbAlc at baseline and 3 months after periodontal therapy. There were no statistically significant difference in periodontal parameters between groups $(P>0.05)$ and no significant improvement in the level of HbAlc in the test group. Microbiological studies indicated that there were significant reductions in the levels of the tested pathogens in both groups. The results of our study were similar to the findings of several other studies that had been done previously.
\end{abstract}

\section{Introduction}

Type 1 diabetes represents approximately 5 to $10 \%$ of all diagnostic cases of diabetes. It is characterized by chronic hyperglycemia caused by autoimmune pancreatic $\beta$-cells destruction generally leading to total loss of insulin secretion [1]. Periodontal disease is a chronic inflammation that affects the tissues surrounding the teeth in response to accumulation of bacterial biofilm on the teeth [2]. It has been established that bacterial pathogens and their products play an essential role in the initiation of the chronic inflammatory process causing damage to periodontal tissues. The host response appears to play a key role in pathogenesis of periodontitis by amplifying the destructive inflammatory process initiated by the bacterial insult [3]. The complex pathogenesis of this disease is further complicated by the coexistence of systemic diseases, such as diabetes, which has the potential to aggravate the manifestations of periodontitis [4]. Periodontal disease and diabetes mellitus belong to a pathologic condition in which both diseases could negatively interfere with each other, constituting a bidirectional relationship with diabetes increasing the risk for periodontitis and periodontal inflammation negatively affecting glycemic control and the progression of vascular complications. Diabetic individuals, both type 1 and type 2, experience a higher incidence of periodontitis and the severity of the disease correlates with the duration of diabetes and glycemic control reflected by glycated hemoglobin (HbAlc) levels in the blood [5]. Type 1 
diabetes has been recognized as an important modifier of periodontal disease $[6,7]$. Numerous factors related to diabetes have been proposed to increase the severity of periodontal disease in diabetic individuals, such as vascular abnormalities, neutrophil dysfunction, nonenzymatic glycosylation, altered collagen metabolism, and altered monocytic response [8].

Conversely, periodontal disease may be a critical factor for worsening glucose intolerance among patients with diabetes [9] and may increase the risk of diabetic complications. Periodontitis may initiate or propagate insulin resistance by enhancing activation of the overall systemic immune response initiated by cytokines [10]. Elevated circulating levels of tumor necrosis factor- $\alpha$ (TNF- $\alpha$ ), interleukin-6 (IL6 ), and high-sensitivity capsular reactive protein (hs-CRP), which can impair insulin resistance and thereby reduce glycemic control, have been shown in a previous study [11]. This way, the control of periodontal disease is necessary for better systemic health in these individuals.

Numerous studies have been published on the effect of periodontal therapy on glycemic control and some of these researchers have found beneficial effect on glycemic control [12-15]. Other studies have demonstrated no significant effect of periodontal therapy on metabolic control $[9,16,17]$. Due to the contradicting findings in the literature, we wanted to evaluate the influence of nonsurgical periodontal therapy on the metabolic control in type 1 diabetes in Malaysian subjects. We also compared the effect of nonsurgical periodontal therapy on clinical parameters of periodontal disease between subjects with type 1 diabetes and nondiabetic subjects and the effect of periodontal therapy on quantity of periodontal pathogens of these subjects.

\section{Material and Methods}

2.1. Sample Size. Sample size was calculated based on expected mean difference in the reduction of $\mathrm{HbAlc}$ in diabetic group of around $0.9-1.0 \%$ [8]; it was calculated that at least 15 patients would be needed to detect this difference with $80 \%$ power and two sided type 1 error of $5 \%$. The basic formula used was

$$
\begin{gathered}
m=\frac{2 *\left[Z_{(1-\alpha / 2)}+Z_{(1-\beta)}\right]^{2}}{\Delta} \\
Z_{(1-\alpha / 2)}=\text { significance level, } \\
Z_{(1-\beta)}=\text { power, } \\
\Delta=\frac{\text { standard difference }}{\text { effective size }}
\end{gathered}
$$

2.2. Study Population. Forty-one of which 20 subjects were type 1 diabetics and with chronic periodontal disease and 21 subjects with only chronic periodontal disease were enrolled into this study. Patients diagnosed with moderate to advanced periodontitis, established from their periodontal parameter status and dental panoramic radiological findings, and agreeable patients who fulfilled the inclusion criteria were included in this study. Ethical clearance for this study was approved by both Faculty of Dentistry ethical committee (reference number DF OP 1304/0019(P)) and Faculty of Medicine ethical committee (reference number 962.22) at the University of Malaya for conducting this clinical study on human individuals. The recruited patients were brought to the Postgraduate Clinic, Faculty of Dentistry, for clinical examination. All subjects were briefed on the aims and method of the study in detail through verbal explanation and patient information sheets were given to all subjects at the beginning of the study. Consent was obtained from all recruited subjects. In the inclusion criteria, all subjects who participated in this study had to have a minimum of 12 natural teeth present and should have been diagnosed with periodontal disease with 5 or more sites of pockets of $\geq 5 \mathrm{~mm}$ and probing attachment level (PAL) $\geq 3 \mathrm{~mm}$ [17]. The age of the subjects was between 20 and 65 years of either gender and subjects of the test group should have been diagnosed with type 1 diabetes mellitus [18]. The following exclusion criteria were considered: subjects who were pregnant or smokers, subjects who had received periodontal treatment within the last 6 months $[13,15]$, and subjects with any history of antibiotics within the last 3 months $[13,15]$ and nonsteroidal anti-inflammatory drug (NSAIDS) use within the last 3 months [9].

2.3. Experimental Design. Examination of periodontal parameters was done for all subjects of both groups at baseline, 2 and 3 months after treatment (Table 2). For the test group, blood samples were collected at baseline and at the end of three months after periodontal treatment for measuring the glycated hemoglobin (HbAlc). Plaque samples were collected from all subjects at baseline and 3 months after periodontal therapy. After recording the periodontal variables, subjects in both groups received oral hygiene instructions and underwent full mouth debridement in a single session using manual instruments (Graceys curette) combined with ultrasonic scaling.

All subjects were recalled at the end of second and third months after treatment and, at each time, the anamnesis was updated and questioned about any changes in medications related to diabetes therapy and alterations in lifestyle.

2.4. Periodontal Examination. After selection of subjects for the study, their dental and medical histories were taken. The subjects were then administered a periodontal clinical examination performed in six sites per tooth (excluding third molars) by a single trained calibrated examiner. The periodontal parameters that were assessed were visible plaque index (VPI) and gingival bleeding index (GBI) [19]. Probing pocket depths (PPD) and probing attachment level (PAL) were evaluated using a Florida probe. Orthopantograms were taken for radiographic assessment of alveolar bone resorption for all the subjects.

2.5. Metabolic Measurement. Blood samples were collected from all subjects in the test group at baseline before treatment and at the end of 3 months after treatment at the University of Malaya Medical Centre and sent to the diagnostic 
laboratory of the Medical Centre for measurement of the concentration level of (HbAlc) (\%), which was measured by high performance liquid chromatography. Standardization of this procedure was achieved by sending all blood specimens to the same diagnostic laboratory.

\subsection{Sample Collection and Bacterial DNA Isolation. After} all supra gingival plaque and calculus were removed using sterile Gracey's curette, sampling sites were isolated using cotton roll. The selected teeth were then air dried and sterile paper point (size 40) was inserted into deepest pocket of each quadrant for 30 seconds. The paper points, 4 in total for each subject, were then packed into microcentrifuge tube containing $1.5 \mathrm{~mL}$ of phosphate buffer solution (PBS). The samples were stored at $-20^{\circ} \mathrm{C}$ until being ready for DNA extraction.

Samples were thawed and vortexed for 10 seconds. After removing the paper points from the tubes, samples were then pelleted by refrigerated centrifuge at $32,000 \mathrm{rpm}$ for 10 minutes at $4^{\circ} \mathrm{C}$. The pellet was used directly for total bacterial genomic DNA extraction using QIAamp DNA Mini Blood Mini Kit (Qiagen, GmBH Germany) according to the manufacturer's instructions. The quality (A260/A280, A260/230) and concentration of the gDNA (ng/ $\mu \mathrm{L})$ were determined using the Nanodrop spectrophotometer ND2000 (NanoDrop Technologies, Wilmington, DE, USA).

2.7. Real Time PCR Detection. The standard curves of $P$. gingivalis (PG), A. actinomycetemcomitans (AA), and $T$. forsythia (TF) were carried out in 6 series of 10 -fold dilutions from $2 \times 10^{5}$ to $2 \times 10^{0}$ according to the manufacturer's instruction (PrimerDesign genesig Kit, Southampton, United Kingdom). The gDNA of each sample was obtained and the quantification of PG, TF, and AA was performed using 7500 Fast Real Time PCR System (Applied Biosystems, Foster City, CA, USA) according to the manufacturer's protocol (PrimerDesign genesig Kit, Southampton, United Kingdom). The reaction mixture for the qPCR was done in a total volume of $20 \mu \mathrm{L}$ consisting of $5 \mu \mathrm{L}$ of genomic DNA $(5 \mathrm{ng} / \mu \mathrm{L})$, $10 \mu \mathrm{L}$ of oasig 2X qPCR Mastermix (PrimerDesign genesig Kit, Southampton, United Kingdom), $1 \mu \mathrm{L}$ of primer/probe mixed assay (FAM reporter), and $4 \mu \mathrm{L}$ of nuclease free water. Quantitative PCR was performed on an ABI 7500 Fast Real Time PCR System (Applied Biosystems, Foster City, CA, USA) using the suggested manufacturer's PCR conditions as follows: initial denaturation at $95^{\circ} \mathrm{C}$ for 15 minutes followed by 50 cycles of denaturation for 10 seconds at $95^{\circ} \mathrm{C}$ and annealing for 60 seconds at $60^{\circ} \mathrm{C}$. The reporter dye (FAM) signal was measured relative to the reference dye (ROX).

2.8. Statistical Analysis. SPSS version 18 (SPSS Inc., Chicago, IL, USA) was used to perform the data analyses. The statistical significance was set for a $P$ value of $<0.05$. The data was tested for normality of distribution using the Shapiro-Wilk test before the test of hypothesis analysis. Mann-Whitney Test was used to analyze the significant difference in age distribution between the test and control group while Chi square test was used to analyze the significant difference in distribution
TABLE 1: Sociodemographic data of study sample.

\begin{tabular}{lccc}
\hline & Test group & Control group & $P$ value \\
\hline Age $($ mean \pm SD) & $37.45 \pm 14.38$ & $44.33 \pm 11.48$ & 0.140 \\
Sex & & & \\
$\quad$ Female & $60 \%$ & $66.7 \%$ & 0.658 \\
$\quad$ Male & $40 \%$ & $33.3 \%$ & \\
Race $\%$ & & & \\
$\quad$ Malay & $2(10 \%)$ & $9(42.9 \%)$ & \\
$\quad$ Chinese & $9(45 \%)$ & $9(42.9 \%)$ & 0.024 \\
$\quad$ Indian & $9(45 \%)$ & $3(14.3 \%)$ & \\
$\begin{array}{l}\text { Duration of diabetes } \\
\text { (mean } \pm \text { SD) }\end{array}$ & & & \\
$\quad<7$ years & $3.12 \pm 2.01$ & & \\
$\quad 7-12$ years & $8.80 \pm 0.83$ & & \\
$\quad<12$ years & $29.0 \pm 8.83$ & & \\
\hline
\end{tabular}

$P$ : $P$ value of comparison between groups.

of gender and ethnicity between the test and control group. Independent Samples Test and Mann-Whitney Test were used to analyze the significant difference between the test and control group before and 3 months after the treatment. Paired Sample $t$-test and Wilcoxon Signed Ranks Test were used to analyze the significant difference in the level of HbAlc, $P$. gingivalis, T. forsythia, and A. actinomycetemcomitans within the same group before and 3 months after the treatment. Repeated Measured ANOVAs were used to analyze the difference in time for each periodontal variable within and between groups.

\section{Results}

The mean age of participants in test and control groups was 37.45 and 44.33 years, respectively, as there was no significant difference between groups in age distribution $(P>0.05)$. For gender distribution, about $40 \%$ of the gender in test group were males while the remaining $60 \%$ were females. In the control group, $33.3 \%$ were males and $66.7 \%$ were females. In the test group the distribution of Chinese and Indians participants was equal, $45.0 \%$ for each ethnic group, while $10 \%$ were Malays and for the control group about 3\% were Indians while Malays and Chinese were $42.9 \%$ and chi square test detected significant difference in distribution of ethnicity between groups $(P<0.05)$. The majority of participants in the test group had been diabetics for more than 12 years (Table 1).

3.1. Clinical Periodontal Response. At the beginning of this study, there was no significant difference in distribution of periodontal parameters between groups.

At 2 months, plaque scores had significantly reduced $(P<$ $0.05)$ from $45.85 \pm 27.39$ to $14.85 \pm 9.37$ in test group and from $32.4 \pm 20.60$ to $13.09 \pm 11.3$ in the control group. While gingival bleeding score for test group reduced from around $36.70 \pm 22.22$ at baseline to $19.35 \pm 10.81$ at 2 months and 
TABLE 2: Comparison of periodontal parameters between diabetic (test group) and nondiabetic (control group) at baseline and 3 months after periodontal therapy.

\begin{tabular}{|c|c|c|c|c|c|}
\hline & Baseline & 2 months & 3 months & $P_{1}$ value & $P_{2}$ value \\
\hline \multicolumn{6}{|l|}{ Plaque score } \\
\hline Test group & $45.85 \pm 27.39$ & $14.85 \pm 9.37$ & $10.80 \pm 10.81$ & 0.000 & \multirow{2}{*}{0.188} \\
\hline Control group & $32.4 \pm 20.60$ & $13.09 \pm 11.3$ & $9.8 \pm 5.86$ & 0.000 & \\
\hline \multicolumn{6}{|l|}{ Bleeding score } \\
\hline Test group & $36.7 \pm 22.22$ & $19.35 \pm 10.81$ & $12.15 \pm 7.68$ & 0.000 & \multirow{2}{*}{0.671} \\
\hline Control group & $34.04 \pm 20.56$ & $17.52 \pm 10.74$ & $11.76 \pm 9.69$ & 0.000 & \\
\hline \multicolumn{6}{|l|}{$\mathrm{PPD}<4 \mathrm{~mm}$} \\
\hline Test group & $79.69 \pm 15.31$ & $95.73 \pm 3.37$ & $97.76 \pm 1.87$ & 0.000 & \multirow{2}{*}{0.109} \\
\hline Control group & $74.96 \pm 11.49$ & $92.69 \pm 5.6$ & $95.7 \pm 4.13$ & 0.000 & \\
\hline \multicolumn{6}{|l|}{ PPD 4-6 mm } \\
\hline Test group & $19.68 \pm 13.80$ & $4.07 \pm 2.74$ & $2.26 \pm 1.82$ & 0.000 & \multirow{2}{*}{0.053} \\
\hline Control group & $27.35 \pm 13.28$ & $6.44 \pm 4.87$ & $4.10 \pm 3.62$ & 0.000 & \\
\hline \multicolumn{6}{|l|}{$\mathrm{PPD}>6 \mathrm{~mm}$} \\
\hline Test group & $0.68 \pm 1.58$ & $0.19 \pm 0.74$ & $0.00 \pm 0.00$ & 0.065 & \multirow{2}{*}{0.168} \\
\hline Control group & $1.78 \pm 2.80$ & $0.32 \pm 0.73$ & $0.20 \pm 0.69$ & 0.006 & \\
\hline \multicolumn{6}{|l|}{$\mathrm{PAL}<4 \mathrm{~mm}$} \\
\hline Test group & $7.53 \pm 9.98$ & $10.83 \pm 13.60$ & $12.34 \pm 14.93$ & 0.007 & \multirow{2}{*}{0.150} \\
\hline Control group & $9.24 \pm 5.5$ & $16.13 \pm 8.18$ & $19.56 \pm 9.94$ & 0.000 & \\
\hline \multicolumn{6}{|l|}{ PAL 4-6 mm } \\
\hline Test group & $26.90 \pm 18.85$ & $9.89 \pm 11.36$ & $7.46 \pm 9.22$ & 0.000 & \multirow{2}{*}{0.075} \\
\hline Control group & $34.38 \pm 10.69$ & $16.35 \pm 10.28$ & $11.97 \pm 10.02$ & 0.000 & \\
\hline \multicolumn{6}{|l|}{$\mathrm{PAL}>6 \mathrm{~mm}$} \\
\hline Test group & $1.85 \pm 3.26$ & $0.78 \pm 1.64$ & $0.54 \pm 1.67$ & 0.012 & \multirow{2}{*}{0.450} \\
\hline Control group & $3.12 \pm 5.77$ & $1.50 \pm 4.15$ & $1.06 \pm 3.29$ & 0.004 & \\
\hline
\end{tabular}

$P_{1}: P$ value of overall changes from baseline to 2 months and to 3 months.

$P_{2}: P$ value of comparison between groups at baseline and 3 months.

from $34.04 \pm 20.56$ basically to $17.52 \pm 10.74$ at 2 months in the control group. These scores were statistically significant $(P<0.05)$ for both groups. At 3 months of follow-up, both indices remained below $13 \%$ in both groups.

$\mathrm{PPD}<4 \mathrm{~mm}$ at baseline was $79.69 \pm 15.31 \mathrm{~mm}$ for the test group and $74.96 \pm 11.49$ for the control group. At 2 months, the mean percentage of sites of PPD $<4 \mathrm{~mm}$ increased in the test group and it further increased at the end of 3 months, which was statistically significant $(P<0.001)$. In the control group, the changes from baseline to 2 months and 3 months were also statistically significant $(P<0.001)$.

At 2 months, sites with PPD between 4 and $6 \mathrm{~mm}$ reduced by more than $79 \%$ in test group and by about $76 \%$ in the control group which was statistically significant $(P<0.001)$ in both groups and it further reduced at 3 months which was again statistically significant $(P<0.001)$.

Mean percentage of PPD of $>6 \mathrm{~mm}$ reduced at 2 and 3 months of follow-up but this reduction was only significant in the control group.

At 2 months and 3 months of follow-up, mean percentage of PAL was significantly reduced in both groups. Comparison between groups was made to detect the difference between test and control groups in the mean percentage of periodontal variable. Statistical tests showed nonsignificant difference between both groups at any point of time.
3.2. Metabolic Response. The result of this study showed lowering in the HbAlc value after nonsurgical periodontal therapy in the test group from $9.24 \pm 2.34$ at baseline to $8.93 \pm$ 2.35 at 3 months of follow-up. However, this reduction was not significant statistically $(P=0.111)$.

3.3. Microbiological Response. At baseline, there was no significant difference in the quantity of $P$. gingivalis, T. forsythia, and $A$. actinomycetemcomitans between test and control groups (Table 3 ).

At the beginning of this study, the quantity of $P$. gingivalis reduced from around $1.4 \times 10^{7} \pm 2.3 \times 10^{7}$ at baseline to $7.2 \times$ $10^{4} \pm 2.7 \times 10^{4}$ at 3 months of follow-up for test group and from $1.8 \times 10^{7} \pm 2.6 \times 10^{7}$ basically to $3.4 \times 10^{6} \pm 9.5 \times 10^{6}$ at 3 months for control group which was statistically significant for both groups.

The quantity of $T$. forsythia at baseline was $2.7 \times 10^{4} \pm$ $2.0 \times 10^{4}, 3.0 \times 10^{4} \pm 2.7 \times 10^{4}$ for test and control groups, respectively. At 3 months, the quantity of this bacterium reduced to $5.2 \times 10^{3} \pm 1.1 \times 10^{4}$ in test group and to $8.2 \times 10^{3} \pm$ $1.6 \times 10^{4}$ in control group. However, the overall reduction was statistically significant in the test and control groups.

The prevalence of $A$. actinomycetemcomitans at baseline for test group and control group was $7.0 \times 10^{3} \pm 1.2 \times 10^{4}$ and 
TABLE 3: Comparison of the quantity of $P$. gingivalis, $T$. forsythia, and A. actinomycetemcomitans between test and control groups at baseline and 3 months of follow-up visits and HbAlc result for test group.

\begin{tabular}{|c|c|c|c|}
\hline & Baseline & 3 months & $P$ value \\
\hline \multicolumn{4}{|l|}{$\mathrm{Pg}$} \\
\hline Test group & $1.4 \times 10^{7} \pm 2.3 \times 10^{7}$ & $7.2 \times 10^{4} \pm 2.7 \times 10^{4}$ & 0.000 \\
\hline Control group & $1.8 \times 10^{7} \pm 2.6 \times 10^{7}$ & $3.4 \times 10^{6} \pm 9.5 \times 10^{6}$ & 0.033 \\
\hline \multicolumn{4}{|l|}{ Tf } \\
\hline Test group & $2.7 \times 10^{4} \pm 2.0 \times 10^{4}$ & $5.2 \times 10^{3} \pm 1.1 \times 10^{4}$ & 0.000 \\
\hline Control group & $3.0 \times 10^{4} \pm 2.4 \times 10^{4}$ & $8.2 \times 10^{3} \pm 1.6 \times 10^{4}$ & 0.001 \\
\hline \multicolumn{4}{|l|}{$\mathrm{Aa}$} \\
\hline Test group & $7.0 \times 10^{3} \pm 1.2 \times 10^{4}$ & $1.3 \times 10^{3} \pm 4.1 \times 10^{3}$ & 0.227 \\
\hline Control group & $4.1 \times 10^{3} \pm 8.5 \times 10^{3}$ & $6.6 \times 10^{3} \pm 1.4 \times 10^{4}$ & 0.526 \\
\hline \multicolumn{4}{|l|}{ HbAlc \% } \\
\hline Test group & $9.24 \pm 2.34$ & $8.93 \pm 2.35$ & 0.111 \\
\hline
\end{tabular}

$P$ : $P$ value of overall changes from baseline to 3 months.

$4.1 \times 10^{3} \pm 8.5 \times 10^{3}$, respectively; however, this difference between test and control group at baseline was statistically nonsignificant $(P>0.738)$. From baseline to 3 months of follow-up visits both groups showed nonstatistical significant reduction in the mean quantity of $A$. actinomycetemcomitans.

\section{Discussion}

This was a cross-sectional interventional study conducted on Malaysian patients with type 1 diabetes. Most of the researches were conducted locally and in other countries mainly used type 2 patients. Thus this study was one of the first investigations to be conducted on type 1 diabetic Malaysian subjects where the effects of nonsurgical periodontal therapy were evaluated.

In this study there was general improvement of all periodontal parameters in both test and control groups after nonsurgical periodontal therapy with no statistically significant difference between groups at any point of time. The results of this study come in agreement with all previous studies [2025], which showed no significance clinical difference between diabetic and nondiabetic patients in response to nonsurgical periodontal therapy. Both test and control groups in this study showed more than $65 \%$ improvement in plaque scores at 3 months of treatment. Similar findings have been reported by a study conducted by Navarro-Sanchez et al. [22]. As to gingival bleeding scores, both test and control groups showed more than 50\% reduction in GBI. Similar finding had been reported in a study conducted by Tervonen et al. [25]. For PPD, there were significant improvements in PPD at the end of 2 months of follow-up in both groups except for PPD of $>6 \mathrm{~mm}$ for test group. Although there was reduction in mean percentage of sites of PPD of $>6 \mathrm{~mm}$, this reduction did not reach significant level statistically. This could be attributed to power of sites of PPD of $>6 \mathrm{~mm}$, which was considered too small in the test group (0.370) compared to control group (0.706). However, a deep remaining probing pocket depth of $\geq 6 \mathrm{~mm}$ at reevaluation phase may not indicate a failure of treatment, as around $75 \%$ of these sites illustrated improvement of $\geq 1 \mathrm{~mm}$ at reevaluation compared to baseline values [26].

This study also indicated that periodontal therapy did not change the level of HbAlc significantly and this result comes in agreement with previous studies $[9,16,17,23$, 27]. Conversely, other studies suggest that the control of periodontal infection improves glycemic control $[12,13,15$, 22]. Factors such as periodontal disease severity, HbAlc monitoring duration, type of diabetes, and the use of local or systemic antibiotics could probably explain some of these differences in the results. The addition of antibiotics, usually the tetracycline family to mechanical debridement, has been demonstrated to have a positive effect not only on clinical periodontal and microbiological parameters but also on metabolic control compared to mechanical treatment alone [28]. A pilot study conducted in nine Hispanics in which the mechanical therapy was combined with the systemic administration of doxycycline demonstrated a $0.6 \%$ reduction in the level of glycated hemoglobin in type 2 diabetic patients [14]. The other factor which we had to consider was the type of diabetes that the subjects had, as most of studies showed significant effect of periodontal therapy on type 2 diabetes mellitus patients [13,29], while other studies carried out on type 1 diabetics have shown no significant effect of periodontal therapy on glycemic control [16, 17, 23]. One possible explanation of our results might be because of the small sample size and it was possible that our small sample size prohibited detection of HbAlc changes. However, Janket et al. [30] had suggested that changes in HbAlc might be less evident in type 1 diabetes, as this disease is due to an autoimmune process and is controlled by insulin administration. These patients are known to maintain a tighter control of their glycemic control.

This study showed significant reductions in the quantity of $P$. gingivalis and $T$. forsythia in both groups following nonsurgical periodontal therapy while the level of $A$. actinomycetemcomitans was unaffected, which is in agreement with previous studies [31-33]. Takamatsu et al. [33] investigated the short-term effects of nonsurgical periodontal therapy on the quantity of $P$. gingivalis and T. forsythia in 26 periodontally 
diseased patients using DNA probe. The prevalence of $A$. actinomycetemcomitans was also identified, but, by using PCR, this study demonstrated significant reduction in the levels of $P$. gingivalis and $T$. forsythia while levels of $A$. actinomycetemcomitans were unaffected.

In this study we also found high levels of both $P$. gingivalis and $T$. forsythia in diseased sites before scaling and root planning compared to healthy sites after therapy and the prevalence of $P$. gingivalis and $T$. forsythia decreased significantly after periodontal therapy. The same results have also been reported in other studies [34]. Many studies evaluating the mean percentage of sites colonized by $T$. forsythia in subgingival plaque have demonstrated significantly higher frequency in diseased patients compared to healthy controls [34]. Moreover, T. forsythia was closely associated with $P$. gingivalis colonization and was more prevalent in the older age groups [35].

In this study, nonsurgical periodontal therapy did not succeed in reducing the level of $A$. actinomycetemcomitans significantly; similar results have also been reported in the previous studies [36] which reported significant improvements in clinical periodontal parameters combined with reduction in the level of $P$. gingivalis following root debridement, while $A$. actinomycetemcomitans was still present in high proportions. Studies have also demonstrated that it is difficult to eradicate A. actinomycetemcomitans from periodontal tissues of aggressive periodontitis by mechanical periodontal therapy only [37], and this could be contributed to the ability of $A$. actinomycetemcomitans to invade deeply into gingival tissue. Furthermore, this study was conducted on subjects with chronic periodontitis, which is mainly caused by red complex species while $A$. actinomycetemcomitans is a microorganism that is highly associated with an aggressive form of periodontal disease found in young adults [38].

Some studies previously also reported that microbiota associated with diabetes does not appear to be different from microbiota of nondiabetics [39]. In this study no significant difference has been reported in the prevalence $P$. gingivalis, T. forsythia, and A. actinomycetemcomitans between diabetic and nondiabetic subjects at baseline and 3 months of followup, except for $A$. actinomycetemcomitans, which increased in the nondiabetic group at 3 months of follow-up. This could possibly be due to reduced compliance to oral hygiene instructions in several participants in the control group, where higher plaque scores were detected from 2 months to 3 months of follow-up.

In this study, we also found that there were no differences in response to nonsurgical periodontal therapy in clinical parameters, microbiological profile, and glycemic control in the three main ethnic groups (Malay, Chinese, and Indian) found in the Malaysian population.

\section{Conclusion}

Both test and control treatments produced similar improvements in clinical parameters from baseline to three months after treatment. However, there were no significant differences between test and control groups at any time. The results obtained from this study appear to demonstrate no statistically significant association between clinical improvements in the periodontal condition and improved metabolic control of diabetes. From the available data, it appears that mechanical periodontal therapy is an important aspect in the management of patients with periodontal disease and should be included as the routine protocol in dealing with diabetics.

However, future studies on the association of patients with type 1 diabetes and chronic periodontitis should be conducted on a larger sample size to establish the association and linkage.

\section{Conflict of Interests}

The authors declare that there is no conflict of interests regarding the publication of this paper.

\section{Acknowledgment}

This study was supported by PG 037-2012B University of Malaya.

\section{References}

[1] American Diabetes Association, "Diagnosis and classification of diabetes mellitus," Diabetes Care, vol. 33, supplement 1, pp. S62-S69, 2010.

[2] J. Kim and S. Amar, "Periodontal disease and systemic conditions: a bidirectional relationship," Odontology, vol. 94, no. 1, pp. 10-21, 2006.

[3] A. R. Kamer, R. G. Craig, A. P. Dasanayake, M. Brys, L. GlodzikSobanska, and M. J. de Leon, "Inflammation and Alzheimer's disease: possible role of periodontal diseases," Alzheimer's and Dementia, vol. 4, no. 4, pp. 242-250, 2008.

[4] A. Monea, T. Mezei, and M. Monea, "The influence of diabetes mellitus on periodontal tissues: a histological study," Romanian Journal of Morphology and Embryology, vol. 53, no. 3, pp. 491495, 2012.

[5] E. Firatli, "The relationship between clinical periodontal status and insulin-dependent diabetes mellitus. Results after 5 years," Journal of Periodontology, vol. 68, no. 2, pp. 136-140, 1997.

[6] A. Meenawat, K. Punn, V. Srivastava, A. S. Meenawat, R. S. Dolas et al., "Periodontal disease and type I diabetes mellitus: associations with glycemic control and complications," Journal of Indian Society of Periodontology, vol. 17, no. 5, pp. 597-600, 2013.

[7] A. Poplawska-Kita, K. Siewko, P. Szpak et al., "Association between type 1 diabetes and periodontal health," Advances in Medical Sciences, vol. 59, no. 1, pp. 126-131, 2014.

[8] B. L. Mealey and T. W. Oates, "Diabetes mellitus and periodontal diseases," Journal of Periodontology, vol. 77, no. 8, pp. 1289-1303, 2006.

[9] F. O. B. Correa, D. Gonçalves, C. M. S. Figueredo, A. S. Bastos, A. Gustafsson, and S. R. P. Orrico, "Effect of periodontal treatment on metabolic control, systemic inflammation and cytokines in patients with type 2 diabetes," Journal of Clinical Periodontology, vol. 37, no. 1, pp. 53-58, 2010.

[10] B. L. Mealey and L. F. Rose, "Diabetes mellitus and inflammatory periodontal diseases," Current Opinion in Endocrinology, Diabetes and Obesity, vol. 15, no. 2, pp. 135-141, 2008. 
[11] G. W. Taylor, B. A. Burt, M. P. Becker et al., "Severe periodontitis and risk for poor glycemic control in patients with non-insulindependent diabetes mellitus," Journal of Periodontology, vol. 67, no. 10, pp. 1085-1093, 1996.

[12] Y. Iwamoto, F. Nishimura, M. Nakagawa et al., "The effect of antimicrobial periodontal treatment on circulating tumor necrosis factor-alpha and glycated hemoglobin level in patients with type 2 diabetes," Journal of Periodontology, vol. 72, no. 6, pp. 774-778, 2001.

[13] M. Kiran, N. Arpak, E. Ünsal, and M. F. Erdoğan, “The effect of improved periodontal health on metabolic control in type 2 diabetes mellitus," Journal of Clinical Periodontology, vol. 32, no. 3, pp. 266-272, 2005.

[14] L. S. Miller, M. A. Manwell, D. Newbold et al., “The relationship between reduction in periodontal inflammation and diabetes control: a report of 9 cases," Journal of Periodontology, vol. 63, no. 10, pp. 843-848, 1992.

[15] S. Singh, V. Kumar, S. Kumar, and A. Subbappa, "The effect of periodontal therapy on the improvement of glycemic control in patients with type 2 diabetes mellitus: a randomized controlled clinical trial," International Journal of Diabetes in Developing Countries, vol. 28, no. 2, pp. 38-44, 2008.

[16] J. P. Aldridge, V. Lester, T. L. Watts, A. Collins, G. Viberti, and R. F. Wilson, "Single-blind studies of the effects of improved periodontal health on metabolic control in type 1 diabetes mellitus," Journal of Clinical Periodontology, vol. 22, no. 4, pp. 271-275, 1995.

[17] F. Llambés, F. Silvestre, A. Hernández-Mijares, R. Guiha, and R. Caffesse, "The effect of periodontal treatment on metabolic control of type 1 diabetes mellitus," Clinical Oral Investigations, vol. 12, no. 4, pp. 337-343, 2008.

[18] K. G. Alberti and P. Z. Zimmet, "Definition, diagnosis and classification of diabetes mellitus and its complications. Part 1: diagnosis and classification of diabetes mellitus provisional report of a WHO consultation," Diabetic Medicine, vol. 15, pp. 539-553, 1998.

[19] J. Ainamo and I. Bay, "Problems and proposals for recording gingivitis and plaque," International Dental Journal, vol. 25, no. 4, pp. 229-235, 1975.

[20] M. Christgau, K.-D. Palitzsch, G. Schmalz, U. Kreiner, and S. Frenzel, "Healing response to non-surgical periodontal therapy in patients with diabetes mellitus: clinical, microbiological, and immunologic results," Journal of Clinical Periodontology, vol. 25, no. 2, pp. 112-124, 1998.

[21] C. J. Gustke, "Treatment of periodontitis in the diabetic patient: A critical review," Journal of Clinical Periodontology, vol. 26, no. 3, pp. 133-137, 1999.

[22] A. B. Navarro-Sanchez, R. Faria-Almeida, and A. BasconesMartinez, "Effect of non-surgical periodontal therapy on clinical and immunological response and glycaemic control in type 2 diabetic patients with moderate periodontitis," Journal of Clinical Periodontology, vol. 34, no. 10, pp. 835-843, 2007.

[23] G. T. Smith, C. J. Greenbaum, B. D. Johnson, and G. R. Persson, "Short-term responses to periodontal therapy in insulindependent diabetic patients," Journal of Periodontology, vol. 67, no. 8, pp. 794-802, 1996.

[24] T. Tervonen and K. Karjalainen, "Periodontal disease related to diabetic status. A pilot study of the response to periodontal therapy in type 1 diabetes," Journal of Clinical Periodontology, vol. 24, no. 7, pp. 505-510, 1997.

[25] T. Tervonen, M. Knuuttila, L. Pohjamo, and H. Nurkkala, "Immediate response to nonsurgical periodontal treatment in subjects with diabetes mellitus.," Journal of Clinical Periodontology, vol. 18, no. 1, pp. 65-68, 1991.

[26] N. Claffey, B. Loos, B. Gantes, M. Martin, and J. Egelberg, "Probing depth at re-evaluation following initial periodontal therapy to indicate the initial response to treatment.," Journal of Clinical Periodontology, vol. 16, no. 4, pp. 229-233, 1989.

[27] J. A. Jones, D. R. Miller, C. J. Wehler et al., "Does periodontal care improve glycemic control? The department of veterans affairs dental diabetes study," Journal of Clinical Periodontology, vol. 34, no. 1, pp. 46-52, 2007.

[28] S. G. Grossi, F. B. Skrepcinski, T. DeCaro et al., "Treatment of periodontal disease in diabetics reduces glycated hemoglobin," Journal of Periodontology, vol. 68, no. 8, pp. 713-719, 1997.

[29] S. Hungund and B. J. Panseriya, "Reduction in HbAlc levels following non-surgical periodontal therapy in type-2 diabetic patients with chronic generalized periodontitis: a periodontist's role," Journal of Indian Society of Periodontology, vol. 16, no. 1, pp. 16-21, 2012.

[30] S. J-. Janket, A. Wightman, A. E. Baird, T. E. van Dyke, and J. A. Jones, "Does periodontal treatment improve glycemic control in diabetic patients? A meta-analysis of intervention studies," Journal of Dental Research, vol. 84, no. 12, pp. 1154-1159, 2005.

[31] A. P. Colombo, R. P. Teles, M. C. Torres et al., "Effects of non-surgical mechanical therapy on the subgingival microbiota of Brazilians with untreated chronic periodontitis: 9-months results," Journal of Periodontology, vol. 76, no. 5, pp. 778-784, 2005.

[32] A. D. Haffajee, "The effect of SRP on the clinical and microbiological parameters of periodontal diseases," Journal of Clinical Periodontology, vol. 24, no. 5, pp. 324-334, 1997.

[33] N. Takamatsu, K. Yano, T. He, M. Umeda, and I. Ishikawa, "Effect of initial periodontal therapy on the frequency of detecting Bacteroides forsythus, Porphyromonas gingivalis, and Actinobacillus actinomycetemcomitans," Journal of Periodontology, vol. 70, no. 6, pp. 574-580, 1999.

[34] A. D. Haffajee, M. A. Cugini, A. Tanner et al., "Subgingival microbiota in healthy, well-maintained elder and periodontitis subjects," Journal of Clinical Periodontology, vol. 25, no. 5, pp. 346-353, 1998.

[35] H. Yang, Y. Huang, and M. Chou, "Occurrence of Porphyromonas gingivalis and Tannerella forsythensis in periodontally diseased and healthy subjects," Journal of Periodontology, vol. 75, no. 8, pp. 1077-1083, 2004.

[36] S. Renvert, M. Wikstrom, G. Dahlen, J. Slots, and J. Egelberg, "Effect of root debridement on the elimination of Actinobacillus actinomycetemcomitans and Bacteroides gingivalis from periodontal pockets," Journal of Clinical Periodontology, vol. 17, no. 6, pp. 345-350, 1990.

[37] W. O. Chung, J. Gabany, G. R. Persson, and M. C. Roberts, "Distribution of erm(F) and tet(Q) genes in 4 oral bacterial species and genotypic variation between resistant and susceptible isolates," Journal of Clinical Periodontology, vol. 29, no. 2, pp. 152-158, 2002.

[38] D. H. Fine, J. B. Kaplan, S. C. Kachlany, and H. C. Schreiner, "How we got attached to Actinobacillus actinomycetemcomitans: a model for infectious diseases," Periodontology 2000, vol. 42, no. 1, pp. 114-157, 2006.

[39] P. P. Katz, M. R. Wirthlin Jr., S. M. Szpunar, J. V. Selby, S. J. Sepe, and J. A. Showstack, "Epidemiology and prevention of periodontal disease in individuals with diabetes," Diabetes Care, vol. 14 , no. 5, pp. 375-385, 1991. 


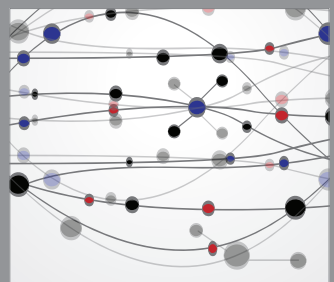

The Scientific World Journal
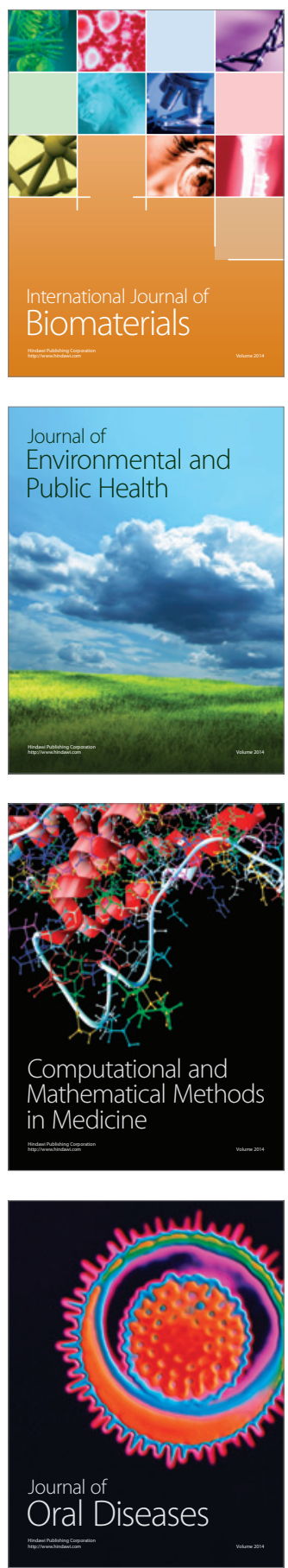
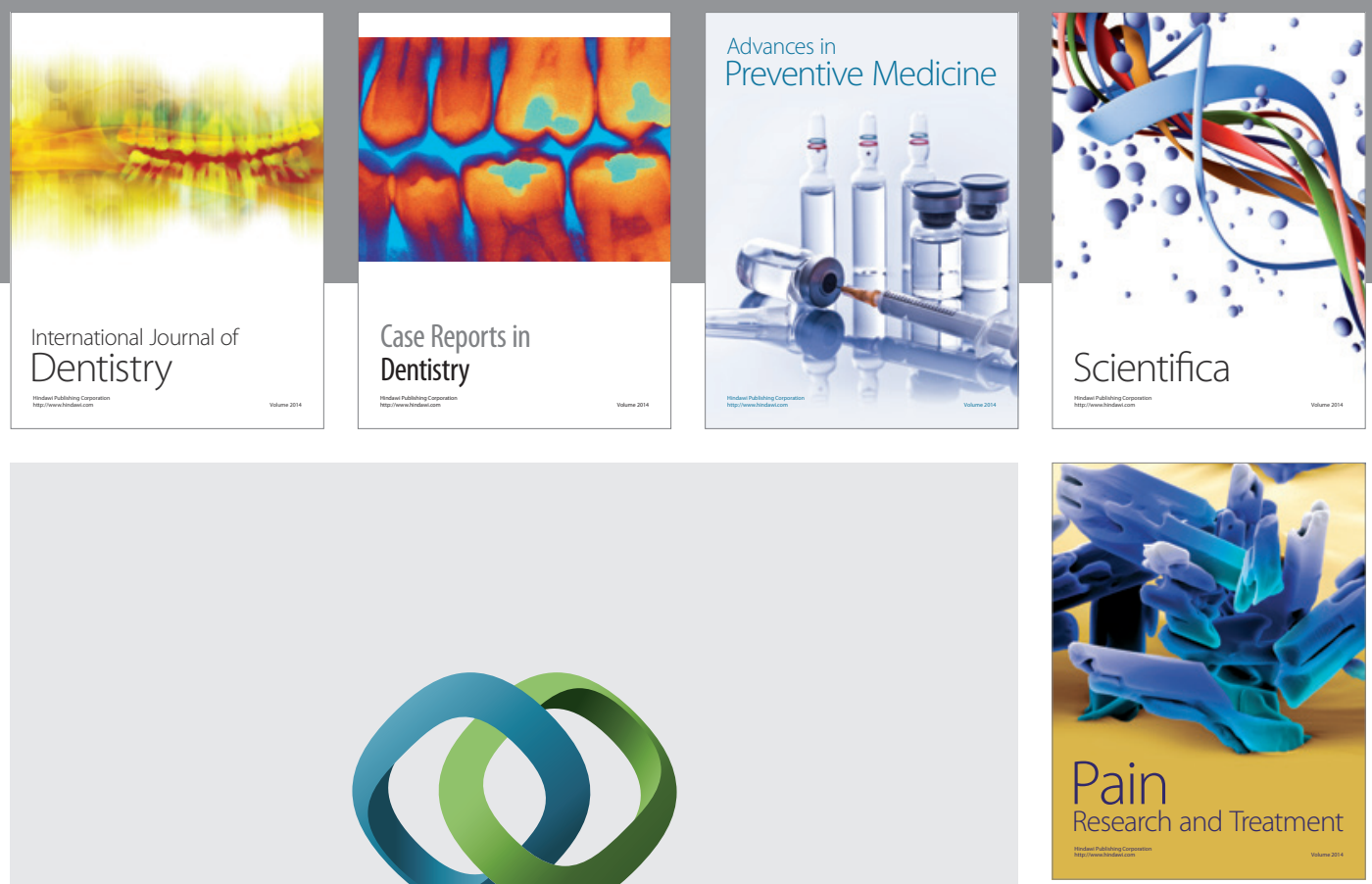

\section{Hindawi}

Submit your manuscripts at

http://www.hindawi.com
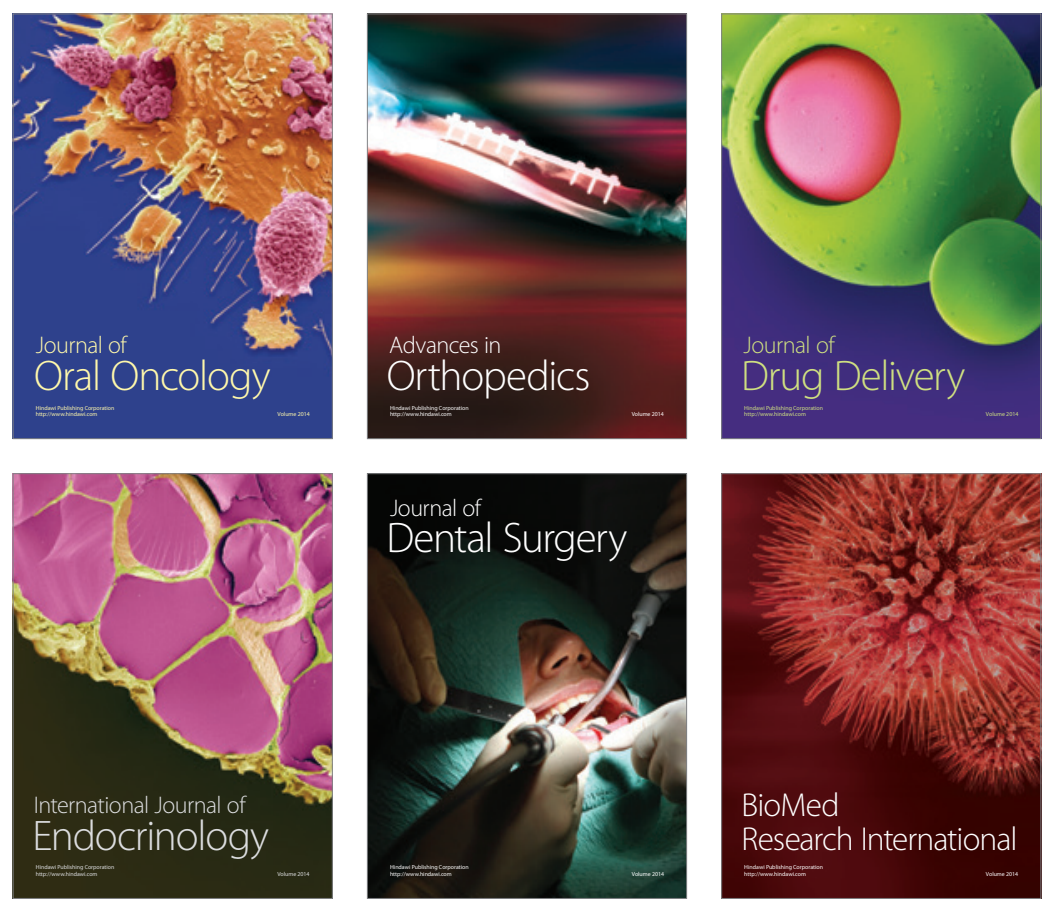

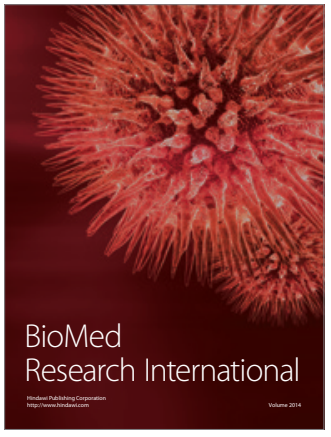

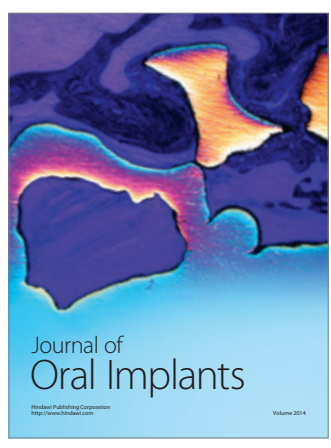
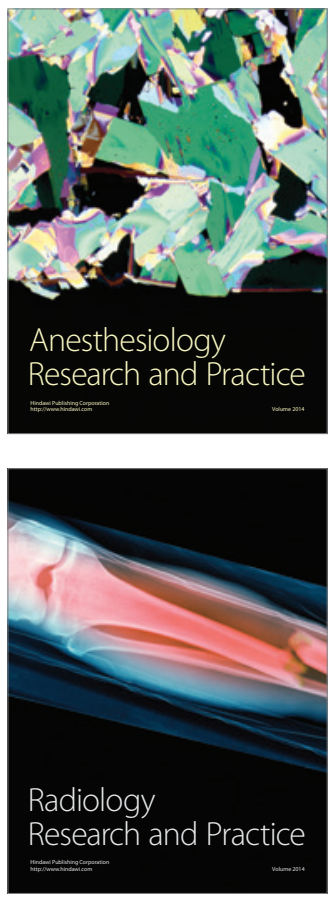